\title{
E quando as outras ciências influenciam as ciências exatas? Ou... Em defesa de um ensino transversal
}

\author{
Nícolas de Araújo Moreira (D)
}

\begin{abstract}
Resumo
O presente artigo visa agregar contribuições à bibliografia do ensino da matemática no Brasil, apresentando aplicações da biologia, estudo das línguas e da geologia às ciências exatas. Tal estudo objetiva motivar o "estudante de exatas" a considerar a importância do estudo das demais disciplinas e entender que as diversas áreas do conhecimento não são isoladas: embora transmitidas como áreas do saber distintas, não são independentes, dialogam entre si. Tal ponto de vista garantirá ao então estudante e futuro profissional uma visão holística do saber que lhe permitirá dialogar com profissionais dos mais diferentes setores. Assim, o presente artigo é um manifesto pelo ensino transversal, multi e interdisciplinar.
\end{abstract}

Palavras-chave: Educação e Ensino de Matemática; Transversalidade no Ensino Médio; Interdisciplinaridade no Ensino Médio.

\begin{abstract}
The present paper aims to give contributions to the bibliography of mathematics teaching in Brazil presenting applications of biology, languages and geology to exact sciences. This study aims to motivate the "student of exact sciences" to consider the importance of studying other disciplines and understand that the diverse fields of knowledge are not isolated: although transmitted as distinct fields of knowledge, they are not independent, they "talk to each other". This point of view will guarantee to the student and future professional a holistic view of knowledge that will allow him to have conversations with professionals from different fields. So, the present paper is a manifest for the transversal teaching, multi and interdisciplinarity.
\end{abstract}

Keywords: Teaching and Mathematics Teaching; Trasversality on High School; Interdisciplinarity on High School.

\section{Introdução: contexto, justificativas e motivação}

É muito frequente que professores em sala de aula sejam confrontados por seus alunos a respeito das aplicações do assunto abordado. Neste caso, é imperativo que o professor, a fim de garantir que os alunos se sintam motivados, tenha alguma familiaridade com aplicações de assuntos como funções exponenciais em modelagem de propagação de doenças infecciosas [1], trigonometria e números complexos aos circuitos de corrente alternada [2], matrizes em otimização de despesas e 
margem de lucro em empresas [3], controle de sistemas dinâmicos [4, 5], modelagem de tráfego em ruas usando grafos [6], sistemas elétricos de potência [7], probabilidades em genética [8], estatística em dados geográficos [9] e de medicina (como em epidemiologia) etc.

Entretanto, poucas vezes são citadas as aplicações das demais disciplinas nas ciências exatas, o que pode acarretar um eventual desinteresse dos estudantes que possuam alguma predileção pelas ciências exatas pelas disciplinas de filosofia e sociologia, história, geografia, língua portuguesa e estrangeiras, biologia, entre outras. Ou seja, estudantes que tenham preferência por matemática, física e química podem acabar por negligenciar as demais matérias por falta do conhecimento da importância que as demais possam vir a ter em suas carreiras, sejam elas quais forem e tal negligência pode causar prejuízos futuros.

Em um âmbito mais geral, a divisão do conhecimento em áreas, realizada por questões didáticas, pode conduzir à visão enganosa de que as subáreas do conhecimento humano são independentes entre si.

Outro ponto importante de se ressaltar é que, uma vez que o mercado de trabalho tem exigido cada vez mais a participação dos trabalhadores em equipes multidisciplinares, é importante que as pessoas tenham domínio do vocabulário e de conceitos básicos de todas as ciências básicas. A percepção deste fato não constitui uma abordagem pedagógica inovadora: diversas universidades francesas como a prestigiosa École Polytechnique e o grupo das Écoles Centrales [10, 11] possuem uma grade curricular com forte base de matemática e física mas que ao mesmo tempo insere disciplinas não só das mais diversas engenharias como também diversas ciências como economia e matemática financeira, administração e empreendedorismo, biologia, filosofia e sociologia, línguas estrangeiras, noções de direito e até mesmo atividades artísticas e educação física como componentes curriculares obrigatórios. O resultado dessa abordagem são engenheiros com forte capacidade de integrar diferentes ideias que permitem soluções inovadoras para problemas complexos. Não à toa, a França é berço de grandes empresas do setor automobilístico e aeroespacial.

\subsection{Objetivos}

O objetivo do presente artigo é apresentar a professores e alunos aplicações das demais áreas do conhecimento nas ciências exatas, mostrando a importância de se estudar todos os temas. Assim, o presente trabalho objetiva, sobretudo, ser um manifesto em defesa do ensino multi e interdisciplinar e da transversalidade, mostrando que as áreas do conhecimento humano não são independentes entre si.

Naturalmente, a filosofia possui uma ligação mais íntima e direta com a matemática por meio da lógica. Entretanto, em outras disciplinas tal ligação é menos óbvia, o que não significa que não tenham menos impacto.

\subsection{Organização do artigo}

O restante do artigo concentrar-se-á em expor aplicações da biologia, das línguas e da geografia na matemática. É importante salientar que a lista não é exaustiva e a maioria das aplicações são mais avançadas e fogem ao escopo do conteúdo abordado no ensino médio. Contudo, a questão central é que o aluno esteja ciente da existência das ligações e das aplicações, objetivando que o aluno estude essas matérias consciente de que, em sua futura vida profissional, eventualmente poderá ter contato com disciplinas do ensino médio que não tenham dado a devida importância e que são, 
sem dúvida, importantes em suas carreiras, independentes de quais sejam. Uma vez apresentadas algumas aplicações, o autor espera que estas sirvam de defesa para a conclusão de que o ensino básico deve ser transversal, multi e interdisciplinar e que evidencie a interdependência dos diversos saberes do conhecimento humano.

\section{Aplicações da biologia}

Cada vez mais a ciência da computação tem se inspirado em modelos biológicos para propor algoritmos que resolvam problemas ligados à otimização, reconhecimento de padrões, entre outros, dando origem a subáreas como a computação neuro inspirada e inteligência artificial. A presente seção irá apresentar algumas dessas aplicações para que se tenha ideia da importância do estudo da biologia - mesmo para os estudantes que pretendam seguir carreira dentro das ciências exatas - por meio de exemplos de aplicações dos conceitos vistos dentro da disciplina de biologia ainda no ensino médio. Dessa forma, espera-se que os professores de ambas as disciplinas realizem intercâmbios de seus conhecimentos entre si para que possam expor aos alunos exemplos que os motivem a estudar as demais disciplinas, como, no caso, a biologia.

\subsection{A biologia do cérebro, redes neurais e reconhecimento de padrões}

Em 1943, W. S. McCullogh e W. Pitts propuseram em [12] um modelo matemático para o neurônio. O neurônio é uma célula nervosa composta por dendritos, corpo celular e axônio. No caso de um neurônio artificial, os dendritos correspondem aos canais de entrada, o corpo celular ao local onde é feita a soma das contribuições correspondentes a cada entrada, e o axônio é representado pelo fluxo de propagação da informação em direção às sinapses. Assim, como em um neurônio real, um neurônio artificial segue o sentido dendritos, corpo celular e axônio [13].

Cada dendrito é modelado como uma entrada $\left(\mathrm{x}_{\mathrm{i}}\right)$ de informação, e a cada entrada é associada um peso sináptico $\left(\mathrm{w}_{\mathrm{i}}\right)$ que mede a eficiência das conexões sinápticas. O corpo celular possui a função de somar as entradas ponderadas pelos seus respectivos pesos sinápticos $\left(\sum_{\mathrm{i}} \mathrm{x}_{\mathrm{i}} \mathrm{w}_{\mathrm{i}}\right)$. Por fim, o axônio determina se houve ou não envio do potencial de ação de acordo com um determinado limiar [13]. Uma comparação entre as estruturas de um neurônio natural e o modelo proposto por McCullogh e Pitts pode ser visto na Figura 1.

Denomina-se perceptron simples a associação de um neurônio de MuCulogh-Pitts a uma regra de aprendizagem. O conceito foi apresentado por Frank Rosenblatt em 1958 em [14].

Uma rede é composta por diversas camadas (em geral camadas de entrada, oculta - responsável pelo processamento não linear desta entrada, e de saída). A forma mais comum de treinamento das redes neurais dá-se pela retropropagação de erro (error backpropagation), que fornece à rede neural a capacidade de "aprender" conforme são fornecidas amostras de treinamento [13]. As redes neurais são arquiteturas essenciais no campo da inteligência artificial, com amplas aplicações em reconhecimentos de padrões, notadamente imagens e áudio, bem como em problemas de interpolação e projeção de dados. A partir desse conceito simples baseado no neurônio natural, um novo conjunto de algoritmos de aprendizagem em machine e deep learning tem sido desenvolvido, como Redes Neurais Convolucionais, Generative Adversarial Networks etc. 


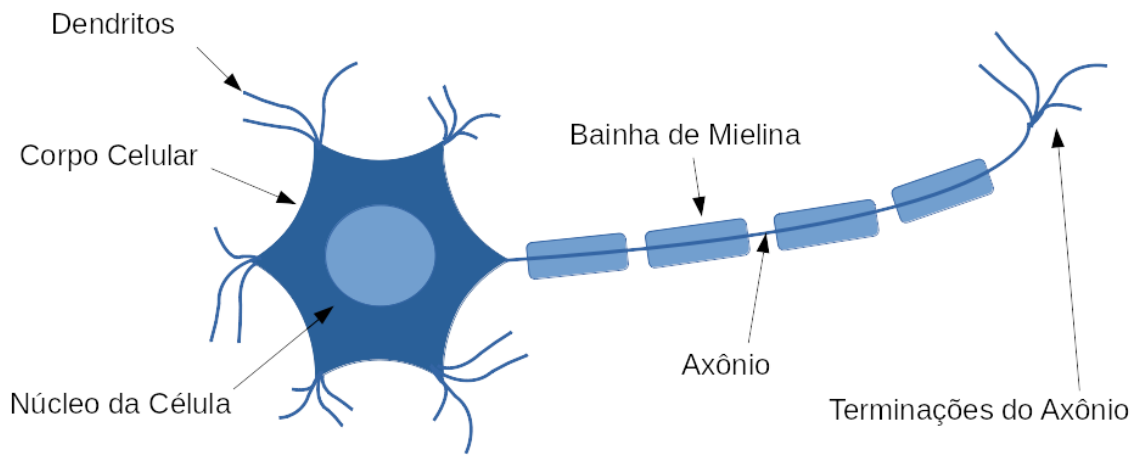

Figura 1: Estrutura de um neurônio convencional.

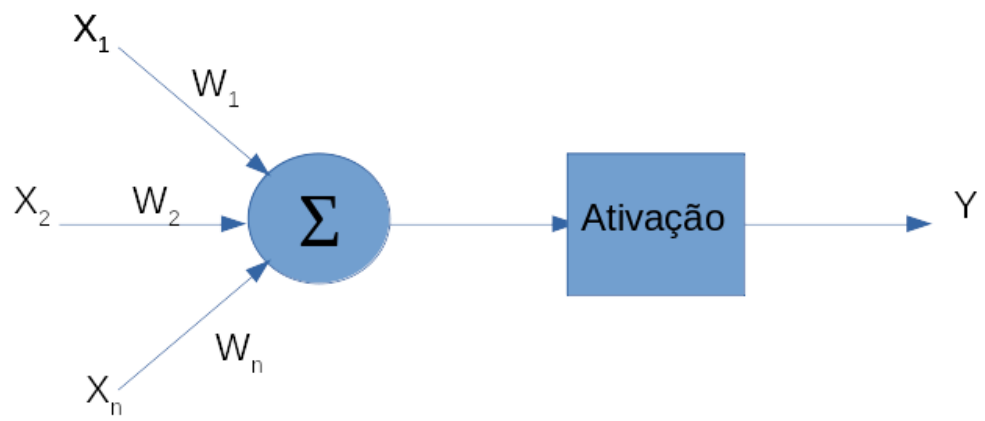

Figura 2: arquitetura proposta por McCullogh e Pitts.

\subsection{Evolução, colônias de formigas e otimização de sistemas}

Define-se algoritmo evolucionário como algoritmo que se inspira nos processos evolutivos como ferramenta para resolver problemas. Tais algoritmos possuem etapas que envolvem seleção, mutação e reprodução: há uma população de estruturas (indivíduos ou cromossomos) sobre a qual se aplicam operadores genéticos de recombinação, mutação (que evita que o algoritmo convirja para um máximo ou mínimo local ao modificar um trecho da informação codificada aleatoriamente) etc. Cada indivíduo é avaliado como solução do problema em questão. Sobre tais indivíduos são aplicados outros operadores genéticos que permitem a seleção (sobrevivência) da melhor solução (solução mais apta). Assim, como podemos ver, os operadores genéticos simulam fenômenos naturais: selecionam-se soluções (pais) que, ao serem recombinadas (aplicação do operador crossover), espera-se que gerem soluções melhores (do contrário tais soluções não "sobreviverão" por muito tempo) até que seja encontrada uma solução ótima para o problema dado. Cada iteração desse processo de selecionar elementos e gerar novos pode ser considerada como uma geração $[15,16]$.

Algoritmos genéticos são uma classe de meta-heurísticas do tipo algoritmos evolucionários voltados para otimização global. Termos como cromossomo, gen, alelo locus, genótipo e fenótipo fazem 
parte do vocabulário e possuem significado no desenvolvimento de algoritmos genéticos, representando respectivamente um indivíduo/string, característica, valor, posição, estrutura e conjunto de parâmetros, respectivamente $[15,16]$.

Em geral, algoritmos genéticos são aplicados para os problemas em que uma solução analítica é desconhecida ou cujos algoritmos existentes demandam um custo/tempo computacional muito grande e cuja diferença entre a solução ótima e a solução subótima encontrada pelo algoritmo genético é negligenciável $[15,16]$.

Outro comportamento da natureza que inspira algoritmos de otimização é o comportamento de colônias de formigas: formigas vagam aleatoriamente em busca de alimento. À medida que percorrem seus caminhos, deixam rastros de feromônio para que possam voltar pelo mesmo percurso bem como servir de referência para que as demais formigas possam segui-las. Naturalmente o feromônio irá se tornar cada vez mais fraco com o passar do tempo, ao menos que sejam reforçadas constantemente: neste caso, é lógico que o caminho mais curto (tempo de trajeto mais curto, ou ainda o caminho mais rápido) terá o rastro de feromônio reforçado mais frequentemente e, consequentemente, atrairá mais formigas que, outrora, percorriam outros caminhos mais longos e com feromônio mais fraco. Tal processo ocorrerá até sobrar apenas o caminho com feromônio mais forte, ou seja, cujo feromônio é reforçado mais constante e frequentemente, e que, por essa lógica, será o caminho mais curto (ótimo) [17].

\subsection{A anatomia e fisiologia do trato vocal, fonética e sistemas de síntese de voz}

Atualmente, a interação homem-máquina vai muito além da interação a partir de mouse e teclado. A interação a partir de comandos de voz e respostas sintetizadas tem se tornado cada vez mais comum, seja por ser uma interação mais natural ao ser humano, seja por comodidade - cenários onde as mãos do usuário estão ocupadas, por exemplo, ou em softwares acessíveis voltados para pessoas com deficiência visual. Isso posto, uma vez que se deseja gerar voz artificial a partir de texto escrito (processo denominado síntese ou ainda TTS - Text-to-Speech), é evidente que para se gerar um modelo matemático e em seguida desenvolver um algoritmo de síntese de voz, fazse necessário compreender como é gerada a voz no trato vocal humano e quais sons podem ser produzidos (fonética). Assim, se desejamos imitar a voz humana, faz-se necessário compreender as estruturas e os mecanismos responsáveis pela fala no corpo humano, ou seja, é necessário um estudo preliminar da anatomia e fisiologia do trato vocal. Da mesma forma que, para se entender os "sons" de uma língua, faz-se necessário estudar a fonética desta. Maiores detalhes podem ser obtidos em [18, 19, 20, 21].

\section{Aplicações do estudo do português: gramáticas, dicionários e compiladores}

O professor Evanildo Bechara em sua gramática [22] define linguagem como "qualquer sistema de signos simbólicos empregados na intercomunicação social para expressar e comunicar ideias e sentimentos, isto é, conteúdos de consciência". O autor ainda em [22] destaca a morfossintaxe como componente central da gramática pura, sendo dividida no estudo da palavra e suas formas (morfologia) e o estudo das combinações materiais - funções sintáticas (sintaxe). Em [22] cita também como subdisciplina autônoma da gramática a lexicologia, isto é, o estudo dos lexemas, ou seja, das unidades linguísticas que possuem significado léxico.

Em ciência da computação, denomina-se compilador o sistema responsável por traduzir um códigofonte de um software escrito em uma determinada linguagem de programação para linguagem de 
máquina (formato que permita ser executado pelo computador) [23].

O processo de compilação é composto por duas etapas: análise (também chamada de front-end) e síntese (também chamada de back-end). A etapa de análise subdivide o código-fonte e efetua uma análise gramatical, gerando, ao final do processo, uma espécie de código intermediário. Tal etapa é responsável por verificar se o código-fonte está sintática e semanticamente correto. A etapa de síntese utiliza o código intermediário e uma tabela de símbolos a fim de gerar o programa objeto [23].

A primeira etapa do processo de compilação é denominada de análise léxica (ou ainda leitura ou scanning), onde são lidos os fluxos que compõem o código-fonte e os agrupa em sequências denominadas lexemas. A etapa seguinte é denominada análise sintática e utiliza a saída da etapa anterior denominados tokens e gera em sua saída uma representação intermediária utilizando uma estrutura de dados denominada árvore de sintaxe, cujos nós representam uma operação e onde cada filho desse nó representa os respectivos argumentos dessas operações. As etapas seguintes fazem uso da estrutura gramatical a fim auxiliar na análise do código para gerar o chamado programa objeto. Uma dessas etapas é a análise semântica, que usa os dados da tabela de símbolos e a árvore de sintaxe para verificar a consistência semântica do código-fonte conforme a definição da linguagem. Uma das fases desse processo é, por exemplo, a verificação de tipo, no qual o compilador checa a compatibilidade entre operadores e operandos [23].

Como se pode perceber, a estrutura de um processo de compilação muito se assemelha em certos aspectos com o estudo de um texto na língua portuguesa, onde há uma série de regras bem definidas a serem seguidas para que o texto seja inteligível para um leitor de língua portuguesa. No caso do programa de computador, esse deve ser perfeitamente claro e lógico para que o computador faça exatamente o que o usuário desejar, uma vez que o computador segue estritamente a lógica que lhe foi determinada por meio do algoritmo.

Assim, uma frase deve ser composta por (na maioria das vezes) um sujeito e predicado, e esses devem ter alguma coerência lógica para que a mensagem possa ser inteligível e se tenha uma comunicação efetiva. Na língua portuguesa, o verbo determina a ação feita pelo (ou a recair sobre o) sujeito. Assim como em uma linguagem de programação, o verbo (o operador) pode exigir um complemento (operando) compatível (do tipo adequado, coerente) com o primeiro. O compilador é responsável por verificar a consistência do código, verificar se há erros de semântica e sintaxe para determinar se o mesmo será válido, isto é, inteligível por parte do computador, além de, evidentemente, atuar como tradutor da linguagem de programação para código binário. Um resumo sobre o processo de compilação pode ser visto na Figura 3.

A diferença entre linguagens de programação e as línguas humanas estão no fato de a primeira dirigir-se a um "interlocutor" desprovido de capacidade de interpretação subjetiva, o que não permite margem para construções como figuras de linguagens, exigindo definições precisas, exatas do que se deseja fazer a partir de um conjunto (vocabulário) de instruções bem mais limitadas que qualquer língua. Pode-se afirmar, portanto, que a linguagem a ser utilizada para desenvolver algoritmos e software é mais exigente quanto à clareza e objetividade: há um rigor, uma exigência de que a instrução que se deseja que o computador realize seja expressa de forma clara, sucinta, codificada, sequencial e lógica. Tais características são exigidas, com exceção talvez dos textos literários, providos de maior liberdade e subjetividade, também em textos argumentativos e outros que não necessariamente instruções, onde não deve haver margem para múltiplas camadas interpretativas. Desta forma, espera-se que esteja claro para o leitor que para um bom domínio da lógica de programação, exige-se uma excelente capacidade comunicativa, isto é, de se fazer entender de forma clara e sem dubiedades, uma habilidade treinada com o estudo da língua portuguesa e de 
redações onde se exige o emprego da norma culta.

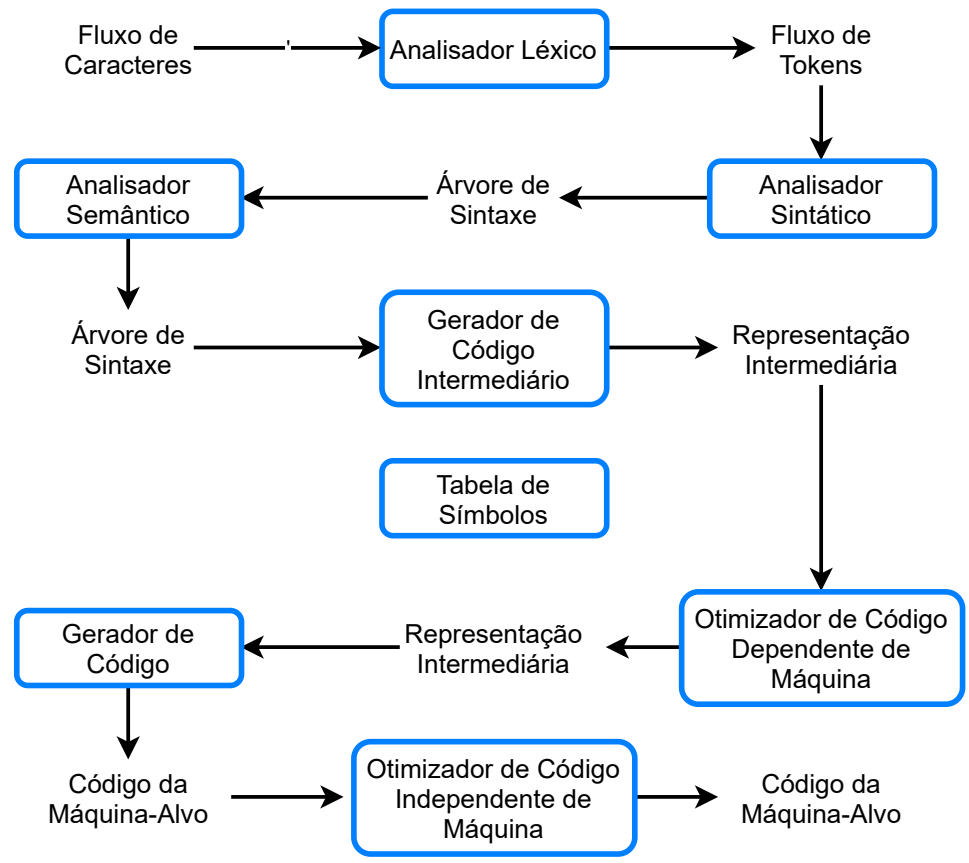

Figura 3: Processos envolvidos na compilação de um código-fonte de programa de computador. Fonte: [23].

\section{De problemas geofísicos a fotos em celulares: a transformada de wavelets}

Na segunda metade dos anos 70, o engenheiro geofísico Jean Morlet da companhia de óleo francesa Elf Acquitaine, graduado pela École Polytechnique em 1952, desenvolveu trabalhos na área da Análise de Wavelets para resolver problemas de processamento de sinais em prospecção de óleo. Suas contribuições, em conjunto com o físico franco-americano de origem croata Alex Grossmann, deram origem à Transformada de Wavelets, que possui inúmeras aplicações, incluindo no formato de compressão de imagens JPEG utilizadas em câmeras fotográficas (de celulares inclusive), que permitem armazenar imagens em alta qualidade ocupando pouca memória, que faz uso da versão discreta da transformada [24, 25].

\section{Conclusão}

Como afirmado inicialmente, estudantes frequentemente questionam seus professores sobre a importância e as aplicações daquilo que estão estudando. O presente artigo visa preencher uma lacuna existente na bibliografia do ensino de matemática a respeito das aplicações das ciências humanas e biológicas nas exatas, apresentado algumas contribuições relevantes do estudo do sistema nervoso central, teoria da evolução, comportamento de manada, anatomia e fisiologia, estudo de gramáticas e dos sismos à matemática, física, computação e engenharias. Com isso, o autor espera 
mostrar que as diversas ciências não são independentes entre si, o que ressalta a importância do ensino transversal, multi e interdisciplinar: não que necessariamente devam ser formados verdadeiros polímatas, mas é essencial que os estudantes do ciclo básico, e se possível, do ensino superior, tenham uma formação básica que cubra ao menos o básico das disciplinas base do conhecimento humano.

Além do objetivo motivacional, que implica maior interesse e consequente foco e atenção nas outras disciplinas, dada a complexidade de projetos e empreendimentos no mundo atual, é importante que o futuro trabalhador da área de exatas seja capaz de dialogar com profissionais das demais áreas. Sendo assim, é importante que os professores de matemática, física e química saibam apresentar exemplos de aplicações e ressaltar a importância dos demais conhecimentos em suas respectivas ciências. Se necessário, o professor deve procurar trocar experiências com colegas engenheiros, que possuam vivência com projetos multidisciplinares ou até mesmo com colegas de áreas mais distintas a fim de enriquecer sua bagagem de aplicações.

Como continuação do presente artigo, o autor sugere e incita os demais colegas a buscarem, apresentarem e divulgarem aplicações da história e da sociologia às ciências exatas, ausentes neste artigo.

\section{Referências}

[1] Gondar, Juan López; Cipolatti, Rolci. Iniciação à física matemática. 2a edição. Rio de Janeiro: Instituto Nacional de Matemática Pura e Aplicada (Impa), 2016.

[2] Nilsson, James W.; Riedel, Susan A. Circuitos elétricos. 8 $8^{\text {a }}$ edição Pearson, 2008.

[3] Puccini, Abelardo de Lima. Programação linear. Rio de Janeiro: LTC, 1989.

[4] Dorf, Richard C.; Bishop, Robert H. Sistemas de controle modernos. 13 ${ }^{\text {a }}$ edição, LTC, 2018.

[5] Ogata, Katsuhiko. Engenharia de controle moderno. $5^{\text {a }}$ edição, Pearson, 2010.

[6] Anton, Howard; Rorres, Chris. Álgebra linear com aplicações. 10 ${ }^{\mathrm{a}}$ edição, Bookman, 2012.

[7] Stevenson Jr., William D. Elementos de análise de sistemas de potência. McGraw-Hill, 1975.

[8] Amabis, José Mariano; Martho, G. R. Fundamentos da biologia moderna - volume único. $4^{\mathrm{a}}$ edição, Moderna, 2006.

[9] Magnoli, Demétrio. Geografia para ensino médio - volume único. 2a edição, Atual Editora, 2012.

[10] École Polytechnique. Cycle d’ingénieur polytechnicien. Disponível em: <https://gargantua. polytechnique.fr/siatel-web/app/linkto/mICYYYTJ(5Z>. Acesso em: 16 de agosto de 2020.

[11] Centrale Supélec. Le Cursus Ingénieur. Disponível em: <https://www.centralesupelec.fr/fr/ le-cursus-ingenieur?tab=pluridisciplinarite-au-coeur-du-cursus $>$. Acesso em: 30 de agosto de 2020.

[12] McCullogh, W.S.; Pitts, W. A logical calculus of the ideas immanent in nervous activity. Bulletin of Mathematical Biophysics, v. 5, pp. 115-133, 1943.

[13] Haykin, Simon. Redes neurais - princípios e práticas. 2a edição. Porto Alegre: Bookman, 2001.

[14] Rosenblat, R. "The perceptron: A probabilistic model for information storage and organization in the brain". Psychological Review, v. 65, pp. 386-408, 1958.

[15] Linden, Ricardo. Algoritmos Genéticos. $2^{\mathrm{a}}$ edição. Rio de Janeiro: Brasport, 2008. 
[16] Viana, Valdísio. Meta-heurísticas e programação paralela em otimização combinatória. Fortaleza: Edições UFC, 1998.

[17] Colorni, A.; Dorigo, M.; Maniezzo, V. "Distributed Optimization by Ant Colonies". Actes de la première conférence européenne sur la vie artificielle, 134-142, Elsevier Publishing, Paris, France, 1991.

[18] Andrade, Eliana X. L.; Sampaio, Rubens; Silva, Geraldo N. Notas em matemática aplicada 22. Sociedade Brasileira de Matemática Aplicada e Computacional. 2006.

[19] Brandão, Alexandre de Souza. Modelagem acústica da produção da voz utilizando técnicas de visualização de imagens médicas associadas a métodos numéricos. Tese de doutorado em Engenharia Mecânica. 172pp. Universidade Federal Fluminense. Niterói, 2011.

[20] Lima, Gislânia Maria de Souza. Modelagem matemática da voz humana: um exemplo de aplicação de um modelo fonte-filtro. Monografia em Física. Universidade Estadual do Ceará. Fortaleza, 2010.

[21] Lopez, Fernando Carrara; Fanganiello, Renato Dalto. Síntese e predição de sinais de voz. Trabalho de Graduação Interdisciplinar em Engenharia Elétrica. Universidade Presbiteriana Mackenzie. 55pp. São Paulo, 2009.

[22] Bechara, Evanildo. Moderna Gramática Portuguesa. 37a edição atualizada pelo Novo Acordo Ortográfico. Rio de Janeiro: Editora Nova Fronteira, 2009.

[23] Aho, Alfred, S. Lam, Monica, Sethi, Ravi, D. Ullman, Jeffrey. Compiladores - princípios, técnicas e ferramentas, $2^{\mathrm{a}}$ edição. São Paulo: Pearson Addison-Wesley, 2008.

[24] Daubechies, Ingrid. Where do wavelets come from? - a personal point of view. Proceedings of the IEEE, v. $84 \mathrm{n}^{\circ}$ 4, pp. 510-513, Institute of Electrical and Electronics Engineers (IEEE), Abril, 1996.

[25] Mallat, Stephane. A Wavelet tour of signal processign - the sparse way. $3^{\mathrm{a}}$ edição. Academic Press, 2009.

Nícolas de Araújo Moreira Universidade Federal do Ceará $<$ nicolas.araujom@gmail.com>

Recebido: 02/09/2020

Publicado: 26/02/2021 